\title{
Tick - Borne Infections as a Cause of Heart Transplantation
}

\author{
ILONA MĄCZKA ${ }^{1}$, TOMASZ CHMIELEWSKI ${ }^{1}$, EWA WALCZAK², JACEK RÓŻAŃSKI ${ }^{3}$, \\ GRZEGORZ RELIGA ${ }^{3}$ and STANISŁAWA TYLEWSKA-WIERZBANOWSKA ${ }^{1}$ \\ ${ }^{1}$ National Institute of Public Health - National Institute of Hygiene, Laboratory of Rickettsia, \\ Chlamydia and Spirochetes, Warsaw, Poland \\ ${ }^{2}$ Institute of Rheumatology, Department of Pathology, Warsaw, Poland \\ ${ }^{3}$ Institute of Cardiology, Warsaw, Poland
}

Received 5 August 2011, revised 10 September 2011, accepted 12 September 2011

\begin{abstract}
Many bacterial species can be a cause of various heart diseases, such as: Borrelia burgdorferi sensu lato, Coxiella burnetii and Bartonella spp. The aim of the present studies was to establish if any tick-borne infections can contribute to serious heart disorders resulting in the need for heart transplantation. Myocardium, aortic and mitral valve samples from hearts removed from patients undergoing heart transplantation were tested. The presence of Bartonella spp., Borrelia afzeli and C. burnetii bacteria in malfunctioning human hearts has been shown. DNA of Bartonella spp., B. burgdorferi and C. burnetii were detected in various parts of tested hearts. DNA of B. afzelii and Bartonella spp. were found in the aortic valves. DNA of C. burnetii was detected in the myocardium. Mixed infections with Bartonella spp. and C. burnetii were also observed. Obtained results indicate that diagnosis of Bartonella spp., B. burgdorferi C. burnetii and Rickettsia spp. infections should be considered in cases of infectious endocarditis with negative blood cultures.
\end{abstract}

Ke y words: infective endocarditis, bartonellosis, Q fever, Lyme boreliosis

Many bacterial species and viruses can be a cause of various heart diseases. The pathogens that trigger these disorders are very often fastidious, uncultured bacteria, such as: Borrelia burgdorferi sensu lato, Coxiella burnetii, Bartonella spp. and Rickettsia spp. (Brouqui and Raoult, 2001). This is the reason why infections with these bacteria are very often undiagnosed. For example it is estimated that over $70 \%$ of infective endocarditis cases with negative blood cultures are related to C. burnetii infections (Chmielewski et al., 2003). The following symptoms: myocarditis, endocarditis, pancarditis, perimyocarditis, dilated cardiomyopathy (DCM), conduction and rhythm disturbances, atherosclerotic, cardiovascular and valvular disease may indicate the bacterial etiology of the disease and detection of significant titers of specific antibodies allows identifying the origin of the disease (Brouqui and Raoult, 2001; Fournier and Raoult, 2003; Million et al., 2010). The aim of the present studies was to establish if any tickborne infections can contribute to serious heart disorders resulting in the need for heart transplantation. Moreover, it should be established whether diagnosis of Bartonella spp., B. burgdorferi, C. burnetii and Rickettsia spp. infections should be considered in every case of infectious endocarditis with negative blood cultures.

In this study 17 fixed in formalin samples of myocardium, 23 of aortic and 22 mitral valve from twenty four hearts removed from patients undergoing heart transplantation, and one frozen endomyocardial biopsy sample were tested. They were collected from 2006 to 2010. Each tissue sample was homogenized. DNA was extracted with the QIAamp Tissue kit (QIAGEN Gmbh, Hilden, Germany) according to the manufacturer's descriptions.

B. burgdorferi DNA was detected with L2 and P1 primers for the 16S rRNA and with OA149 and OA319 specific primers for the OspA gene fragments characteristic for all Borrelia species: B. burgdorferi sensu stricto, B. afzelii and B.garinii (Million et al., 2010; Podsiadly et al., 2003). The primers BhCS.781p and BhCS.1137n were used to amplify a 400-bp fragment of the Bartonella spp. citrate synthase gene (Nilsson et al., 2005). Detection of Rickettsia spp. DNA was performed using primers RpCS.409d and RpCS.1258n for conserved regions of the citrate synthase gene (Nocton et al., 1994). C. burnetii DNA was detected with primers

* Corresponding author: I. Mączka, National Institute of Public Health, National Institute of Hygiene, Chocimska 24 Street, 00-791 Warsaw, Poland; phone: (48) 2254212 50; e-mail: imaczka@pzh.gov.pl 
Table I

Primers and nucleotide sequences used in the study

\begin{tabular}{|l|l|l|l|l|}
\hline \multicolumn{1}{|c|}{ Pathogen } & \multicolumn{1}{|c|}{ Primers } & Fragment gene (size, bp) & \multicolumn{1}{c|}{ Nucleotide sequences } & \multicolumn{1}{c|}{ Literature } \\
\hline Rickettsia spp. & RpCS.409d & Citrate synthase gene & 5'-CCTATGGCTATTATGCTTGC & Roux et al. 1997 \\
& RpCS.1258n & (850) & 5'-ATTGCAAAAAGTACAGTGAACA & \\
\hline Borrelia burgdorferi & L2 & 16S rRNA (600) & 5'-GGTCAAGACTGACGCTGAGT & Chmielewski et al. 2003 \\
& P1 & & 5'-TCGCTTTGTACAGGCCATTG & \\
\cline { 2 - 6 } & OA149 & OspA gene & 5'-TTATGAAAAAATATTTATTGGGAAT & Nocton et al. 1994 \\
& OA319 & 5'-CTTTAAGCTCAAGCTTGTCTACTGT & \\
\hline Bartonella spp. & BhCS.781p & citrate synthase gene (400) & 5'-GGGGACCAGCTCATGGTGG & Podsiadly et al. 2007 \\
& BhCS.1137n & & 5'-AATGCAAAAAGAACAGTAAACA & \\
\hline
\end{tabular}

isIIIIf and isIIIIr for the htpAB gene fragment (Palecek et al., 2010). (Table I).

The $50 \mu$ reaction mixtures contained $10 \mathrm{mM}$ Tris$\mathrm{HCl}, 50 \mathrm{mM} \mathrm{KCl}, 2.5 \mathrm{mM} \mathrm{MgCl}_{2}, 0.1 \%$ gelatin, $200 \mu \mathrm{M}$ dNTPs, 50 pmol of each primer and $1.5 \mathrm{U}$ Taq DNA polymerase (Perkin-Elmer Cetus, USA). An aliquot of $5 \mu$ of extracted DNA template was added to each reaction mixture. Each PCR test included negative (water) and positive controls containing DNA of B. afzelii, B. garini, B.henselae, C. burnetii Henzerling strain, Rickettsia conori $\mathrm{H} 24$ strain, from the collection of the NIH. For B. burgdorferi and Rickettsia spp. the cycling conditions were as follows: $3 \mathrm{~min}$ at $95^{\circ} \mathrm{C}$, followed by 40 cycles of $1 \mathrm{~min}$ denaturation at $95^{\circ} \mathrm{C}$, annealing $1 \mathrm{~min}$ at $55^{\circ} \mathrm{C}$, elongation $1 \mathrm{~min}$ at $72^{\circ} \mathrm{C}$ and final elongation $7 \mathrm{~min}$ at $95^{\circ} \mathrm{C}$. For Bartonella spp. the cycling conditions were as follows: $10 \mathrm{~min}$ at $94^{\circ} \mathrm{C}$, followed by 45 cycles of $1 \mathrm{~min}$ at $94^{\circ} \mathrm{C}, 1 \mathrm{~min}$ at $57^{\circ} \mathrm{C}, 2 \mathrm{~min}$ at $72^{\circ} \mathrm{C}$ and finally $10 \mathrm{~min}$ at $72^{\circ} \mathrm{C}$. For C. burnetii the cycling conditions were as follows: $3 \mathrm{~min}$ at $95^{\circ} \mathrm{C}$, followed by 40 cycles of $1 \mathrm{~min}$ at $95^{\circ} \mathrm{C}, 1 \mathrm{~min}$ at $57^{\circ} \mathrm{C}, 1 \mathrm{~min}$ at $72^{\circ} \mathrm{C}$ and finally $7 \mathrm{~min}$ at $95^{\circ} \mathrm{C}$. PCRs were performed in a thermal Mastercycler ep (EP Gradient, Eppendorf AG, Hamburg, Germany). Isolated DNA from strains collected in the National Institute of Public Health - National Institute of Hygiene including: Salmonella thadar, Escherichia coli, Pseudomonas aeruginosa, Shigella flexneri, Yersinia enterocolitica, Leptospira biflexa and Treponema pallidum (kindly provided from Institute of Venerology, Medical University of Warsaw) were used as a negative controls. All amplicons were analyzed in electrophoresis in 1.5\% agarose gel stained with ethidium bromide.

All PCR products were purified with QIAquick PCR purification kit (QIAGEN Gmbh, Hilden, Germany) according to the manufacturer's protocol with one modification. To elute DNA, deionized water instead of EB buffer (10 mM Tris-Cl, pH 8.5) was used. The specificity of all positive results was confirmed by sequencing the amplicons with the ABI 377 DNA Analyzer (Applied Biosystem, USA) according to the manufacturer's recommendations. All sequences were edited using Autoassembler software (Applied Biosystem, USA) and identified using the BLAST software by comparison with sequences available in GenBank.

DNA of Bartonella spp., B. burgdorferi and C. burnetii was detected in various parts of the hearts of patients undergoing heart transplantation. The study group consisted of 4 patients suffered from severe dilated cardiomyopathy of different etiology. DNA of Borrelia afzelii was found in the aortic valves of two patients (B. afzelii 16S rRNA gene, B. afzelii OspA gene). Mixed infections were found in two other patients. In the first, DNA characteristic for Bartonella spp. was detected in the aortic valves and DNA of C. burnetii in the myocardium. In the second patient, DNA characteristic of Bartonella spp. was detected in the mitral valve and DNA of C. burnetii was detected in the myocardium and the aortic valves (Table II). DNA of Rickettsia spp. was not found in any tested material.

Two sequences of is 1111 gene partially sequenced over a total 74 nucleotide positions showed 98\% (myocardium sample) and 100\% (myocardium and aortic valve samples from the same patient) nucleotide identity between detected strains and C. burnetii strains: CbuK_Q154 (Accession number CP001020.1), CbuG_Q212 (CP001019.1), RSA331 (CP000890.1) The sequence of $16 \mathrm{~S}$ rRNA gene partially sequenced over a total 427 nucleotide positions showed 98\% (aortic valve) nucleotide identity between detected strains and B.afzelii strains: Nov1105 (Accession number EF541175.1), Nov11506 (EF541174.1), Mng 3602 (DQ469888.1). One sequence of OspA gene partially sequenced over a total 147 nucleotide positions showed 99\% (aortic valve) nucleotide identity between detected strains and B.afzelii strains: ACA-1 plasmid lp54 (Accession number CP001247.1), PKo plasmid lp60 (CP000396.1). One sequence of citrate synthase (gltA) 
Table II

Location of Borrelia afzelli, Bartonella spp. and Coxiella burnetii DNA in removed hearts of transplant recipients suffering from dilated cardiomyopathy

\begin{tabular}{|c|l|l|l|l|l|}
\hline No. patients & Clinical sample & Borrelia afzelli & Bartonella spp. & Coxiella burnetii & Clinical symptoms \\
\hline 1 & Aortic valve & positive & negative & negative & coronary artery disease \\
\hline 2 & Aortic valve & positive & negative & negative & coronary artery disease \\
\hline 3 & Myocardium & negative & negative & positive & \multirow{2}{*}{ coronary artery disease, aortic stenosis } \\
\cline { 2 - 5 } & Aortic valve & negative & negative & positive & \\
\cline { 2 - 5 } & Mitral valve & negative & positive & negative & \\
\hline \multirow{2}{*}{4} & Myocardium & negative & negative & positive & \multirow{2}{*}{ myocarditis } \\
\cline { 2 - 4 } & Aortic valve & negative & positive & negative & \\
\hline
\end{tabular}

* Bacterial DNA detected with polymerase chain reaction (PCR)

** Bacterial DNA not detected

Rickettsia spp. DNA was not detected

gene partially sequenced over a total 235 nucleotide positions showed 99\% (aortic valve) nucleotide identity between the detected strains and Bartonella sp. strains: clone G10 citrate synthase (gltA) gene (Accession number HM116785.1), BCF02 citrate synthase (gltA) gene (GU056189.1), Sr3sk (AY587980.1). One sequence of citrate synthase (gltA) gene partially sequenced over a total 233 nucleotide positions showed 99\% (mitral valve) nucleotide identity between detected strains and Bartonella sp. strains: clone G10 (Accession number HM116785.1).

Our research has shown the presence of Bartonella spp., B. afzeli and C. burnetii bacteria in malfunctioning human hearts. The detected pathogens, occurring in ticks in the natural environment, have a clinical importance in cardiology (Brouqui and Raoult, 2001; Nilsson et al., 2005). It is well known that C. burnetii, an etiologic agent of $\mathrm{Q}$ fever, is responsible for difficult to cure endocarditis, with high mortality rate (Chmielewski et al., 2003). Generally, C. burnetii infections are located on heart valves. In the presented study the bacteria have also been found in the myocardium. This confirms the rarely cases described when the pathogen is responsible for myocarditis. In the last decade of the twentieth century heart failures due to B. burgdorferi and Bartonella spp. were described. Dilated cardiomyopathy (DCM), conduction and rhythm disturbances have been observed in Lyme borreliosis patients, whereas endocarditis and myocarditis in bartonellosis patients (Brouqui and Raoult, 2001; Podsiadly et al., 2007). The obtained results indicate that among patients with cardiac diseases, infections caused by B. burgdorferi, C. burnetii and Bartonella spp., should be obligatorily tested for. Our results indicate that father studies are needed. It is necessary to exam if the detected bacteria are the direct cause of cardiologic complications or they accompany the other decisive factors.

\section{Acknowledgments}

The research has been supported in part by Ministry of Science and Higher Education [grant No. N N404 3304 33] and NIPH-NIH funds. The authors declare no conflicts of interest.

\section{Literature}

Brouqui P. and D. Raoult. 2001. Endocarditis due to rare and fastidious bacteria. Clin. Microbiol. Rev. 14: 177-207.

Chmielewski T., J. Fiett, M. Gniadkowski and S. Tylewska-Wierzbanowska. 2003. Improvement to laboratory recognition of Lyme borreliosis with the combination of culture and PCR methods. Mol. Diagn. 7: 155-162.

Fournier P.E. and D. Raoult. 2003. Comparison of PCR and serology assays for early diagnosis of acute Q fever. J. Clin. Microbiol. 41: 5094-8.

Million M., F. Thuny, H. Richet and D. Raoult. 2010. Long-term outcome of Q fever endocarditis: a 26-year personal survey. Lancet. Infect. Dis. 10: 527-535.

Nilsson K., A. Liua, C. Pahlsonc and O. Lindquistd. 2005. Demonstration of intracellular microorganisms (Rickettsia spp., Chlamydia pneumoniae, Bartonella spp.) in pathological human aortic valves by PCR. J. Infect. 50: 46-52.

Nocton J.J., F. Dressler, B.J. Rutledge, P.N. Rys, D.H. Persing and A.C. Steere. 1994. Detection of Borrelia burgdorferi DNA by polymerase chain reaction in synovial fluid from patients with Lyme arthritis. N. Engl. J. Med. 330: 229-234.

Palecek T., P. Kuchynka, D. Hulinska, J. Schramlova, H. Hrbackova, I. Vitkova, S. Simek, J. Horak, W.E. Louch and A. Linhart. 2010. Presence of Borrelia burgdorferi in endomyocardial biopsies in patients with new-onset unexplained dilated cardiomyopathy. Med. Microbiol. Immunol. 199: 139-143.

Podsiadly E., T. Chmielewski, R. Marczak, E. Sochon and S. Tylewska-Wierzbanowska. 2007. Bartonella henselae in the human environment in Poland. Scand. J. Infect. Dis. 39: 956-62.

Podsiadły E., T. Chmielewski and S. Tylewska-Wierzbanowska. 2003. Bartonella henselae and Borrelia burgdorferi infections of the central nervous system. Ann. N. Y. Acad. Sci. 990: 404-6.

Roux V., E. Rydkina, M. Eremeeva and D. Raoult. 1997. Citrate synthase gene comparison, a new tool for phylogenetic analysis, and its application for the rickettsiae. Int. J. Syst. Bacteriol. 47: $252-61$. 